\title{
SEEDING to enable sensitive electrochemical detection of biomarkers in undiluted biological samples
}

Jonathan Sabaté del Río

https://orcid.org/0000-0002-3295-5851

Hyun-Kyung Woo

Center for Soft and Living Matter, Institute for Basic Science (IBS), Department of Biomedical

Engineering, Ulsan National Institute of Science and Technology

Juhee Park

IBS https://orcid.org/0000-0002-8109-6387

\section{Hong Koo Ha}

Pusan National University Hospital, Pusan National University School of Medicine

\section{Jae-Ryong Kim}

4Department of Biochemistry and Molecular Biology, Smart-aging Convergence Research Center, College of Medicine, Yeungnam University

Yoon-Kyoung Cho ( $\nabla$ ykcho@unist.ac.kr)

IBS https://orcid.org/0000-0001-6423-1834

\section{Article}

Keywords: Nanostructure, nanoporous gold, surfactant, extracellular vesicle, electrochemistry, biosensor

Posted Date: October 8th, 2021

DOl: https://doi.org/10.21203/rs.3.rs-945043/v1

License: (c) (1) This work is licensed under a Creative Commons Attribution 4.0 International License. Read Full License

Version of Record: A version of this preprint was published at Advanced Materials on April 15th, 2022. See the published version at https://doi.org/10.1002/adma.202200981. 


\section{SEEDING to enable sensitive electrochemical detection of}

\section{2 biomarkers in undiluted biological samples}

3

4 Jonathan Sabaté del Río ${ }^{1}$, Hyun-Kyung Woo ${ }^{1,2}$, Juhee Park ${ }^{1}$, Hong Koo Ha ${ }^{3}$, Jae-

5 Ryong Kim ${ }^{4}$, Yoon-Kyoung $\mathrm{ChO}^{1,2^{*}}$

$6 \quad{ }^{1}$ Center for Soft and Living Matter, Institute for Basic Science (IBS), Ulsan, 44919

7 Republic of Korea; ${ }^{2}$ Department of Biomedical Engineering, Ulsan National Institute of

8 Science and Technology (UNIST), Ulsan, 44919 Republic of Korea; ${ }^{3}$ Department of

9 Urology, Pusan National University Hospital, Pusan National University School of

10 Medicine, Busan 49241, Republic of Korea; ${ }^{4}$ Department of Biochemistry and

11 Molecular Biology, Smart-aging Convergence Research Center, College of Medicine,

12 Yeungnam University, Daegu 42415, Republic of Korea;

13

$14{ }^{*}$ Correspondence to: ykcho@unist.ac.kr (Y.-K.C.) 


\section{Abstract}

17 The interface between an electrode and a liquid plays a critical role in the overall

18 performance of electrochemical biosensors. Surface morphology and

19 roughness affect key parameters, such as the active area, diffusion profiles, and

20 apparent electron transfer kinetics, whereas porosity may hinder the diffusion

21 of fouling proteins. However, there is no simple and rapid method compatible

22 with photolithographic electrodes to generate both nanostructured and porous

23 surfaces. Herein, we demonstrate the interplay between the preferential etching

24 of chloride and surfactant-assisted anisotropic gold reduction to create 25 homogeneous, nanostructured, and nanoporous substrates on

26 photolithographic gold electrodes within a minute and without using templates.

27 We coined this process, SEEDING, that is, Surfactant-based Electrochemical

28 Etch-Deposit Interplay for Nanostructure/Nanopore Growth. SEEDING on 29 electrodes enhanced the sensitivity and anti-biofouling capabilities, enabling 30 direct analysis of small molecules, proteins, and cancer-derived extracellular 31 vesicles in complex biological fluids such as undiluted plasma and urine 32 samples.

34 Keywords: Nanostructure, nanoporous gold, surfactant, extracellular vesicle, 35 electrochemistry, biosensor 
Compact and affordable analytical tools that can rapidly perform sensitive, selective, and multiplexed detection of biological markers are essential for disease diagnostics and implantable sensors. Electrochemical readout platforms present an affordable and sensitive approach, along with miniaturised and simple instrumentation, promoting the fabrication and generalisation of point-of-care medical devices. However, the application of biosensors in diagnostic platforms still faces limitations when measuring relevant clinical samples typically comprising complex biological fluids, such as plasma, because of surface inactivation and sensitivity loss from nonspecific adsorption and accumulation of sample proteins.

Sampling and molecular analysis of human biofluids hold great promise for cancer diagnosis, prognosis, and treatment response assessment ${ }^{1}$. Extracellular vesicles (EVs) secreted by all cells are present in large numbers in nearly all body fluids and contain invaluable protein and genetic information, thereby playing an essential role in various physiological and pathological processes ${ }^{2}$. Therefore, EVs are potential biomarker candidates for liquid biopsy analyses; for instance, tumour-derived EVs (tEVs) are critically related to tumour progression, metastatic niche formation, and immune evasion ${ }^{3,4}$. However, despite their large concentration in plasma, tEVs still represent a minority of all EVs in the body. Additionally, EVs still represent a minor sub-population of all circulating particles in the blood and are outnumbered by lipoproteins by up to six orders of magnitude ${ }^{5}$. Therefore, conventional analysis methods typically require EVs separation, concentration, and purification steps which significantly affecting analysis time and sample volume requirement ${ }^{6}$.

Studies on the detection of tEVs from plasma or serum have mainly been achieved using optical methods ${ }^{7,8}$. However, the high sensitivity required, along with the large 61 concentration of contaminants in clinical samples, impose a compromise between 
sensitivity and selectivity in electrochemical biosensors ${ }^{9}$. A sensitive electrochemical

biosensor with anti-biofouling capabilities may address these requirements providing

a high-throughput tool for tEV diagnostics of small-volume liquid biopsies.

The sensitivity of electrochemical biosensors can be enhanced by increasing their surface $a r e a^{10}$, thereby lowering their limit of detection ${ }^{11}$ and yielding enhanced

67 apparent electron transfer kinetics and improved bioreceptor functionalisation yield ${ }^{12}$. However, a large available surface area also increases biofouling, the non-specific adsorption of sample proteins and molecules, with a subsequent decrease in sensitivity. In this regard, the formation of nanoporous structures may be beneficial for

71 limiting the diffusion of undesired fouling proteins ${ }^{9,13-15}$, although the same principle 72 limits the detection of desired analytes ${ }^{16,17 .}$

73 In this study, we present SEEDING, that is, Surfactant-based Electrochemical

74 Etch-Deposit Interplay for Nanostructure/Nanopore Growth, a method to fabricate gold 75 electrodes that combine nanostructured surfaces and nanoporous substrates, 76 achieving the benefits of both strategies, namely, large available electroactive areas 77 and limited diffusion and adsorption of non-specific proteins. As proof of concept, we 78 designed selective biosensors to detect electroactive molecules, an inflammatory 79 marker interleukin 6 (IL6) protein, and Epithelial Cell Adhesion Molecule $80(\text { EpCAM })^{+}$EVs from undiluted biological samples, such as cell culture media, blood 81 plasma, and urine. The process is a simple, cost-efficient, and rapid electrochemical 82 method that can be conducted on photolithographic chips for mass production of 83 disposable assay chips typically used in portable or handheld devices. 


\section{surfaces}

Application of specific electric potentials to an electrode can transfer electrons from and to nearby molecules in solution, or atoms from the electrode, thereby changing the oxidation state of the species involved. For instance, we can electrooxidise the surface of the electrode to etch materials and/or electrodeposit soluble materials on the electrode. By repeating this process, we can gradually transform a flat electrode into a nanostructured and nanoporous gold (NSG) electrode (Fig. 1a). Electrochemistry provides a convenient tool for controlling the kinetics and thermodynamics of these reactions. However, it is essential for this etch-deposit process to be anisotropic to create NSG electrodes. In other words, the interplay between the etch and deposit process must be balanced and exhibit spatial preference.

97 Otherwise, each process would simply cancel each other, etching gold where it was deposited previously, leading to simple surface roughening ${ }^{18}$.

To achieve controlled and directed growth, we designed the SEEDING method.

We used a sodium chloride solution for electrooxidising and etching gold from a flat electrode and chloroauric acid and cetyltrimethylammonium $\left(\mathrm{CTA}^{+}\right)$surfactant for preferential growth orientation during electroreduction. By quickly repeating these steps, we see that the anodic and cathodic currents, representing the oxidation and reduction of gold, increase over time reaching a stable current within a minute (Fig.

1b, Supplementary Fig. 1). This current is related with the available electroactive area, indicating growth of nanostructures, a process not observed using control solutions containing only one or two of the three components.

The fabricated NSG electrodes exhibited a distinctive dark homogeneous coloration on the surface (Fig. 1c). At the microscopic level, the original gold surface 
comprised polycrystalline gold grains. Grain boundaries were preferential etching sites that would create large crevasses and eventually act as nucleation points where gold would be preferentially deposited. The deposits grow densely and eventually have a coral reef shape with $\sim 20 \mathrm{~nm}$ grain aggregates (Fig. 1d, Supplementary Fig. 2) spanning homogeneously across the entire electrode surface (Supplementary Fig. 3). The root-mean-square roughness measured by atomic force microscopy (AFM) can reach values of $340 \pm 90 \mathrm{~nm}$ for NSG vs. the flat gold $(1.2 \pm 0.3 \mathrm{~nm})$ (Fig. 1e), which leads to a hydrophobic character because of the extreme roughness (Fig. 1f). If we conduct the same process by scanning the voltage on the electrode within a potential window, the process can be analysed in more detail (Fig. 2). Initially, a solution of $\mathrm{CTA}^{+}$in contact with a gold surface forms a compact and homogeneous bilayer that passivates the electrode for direct reduction of gold (Fig. 2-(1)), and CTA in solution aggregate in micelles that coordinate with free chloroauric acid ${ }^{19}$. However, when poising the electrode at anodic potential, chloride can diffuse toward the gold surface $^{20}$ generating compact chloride adatoms at preferential sites ${ }^{21}$ (Fig. 2-(2)), during which both $\mathrm{CTA}^{+}$and chloride adlayers are co-adsorbed on the electrode ${ }^{22,23}$. When the onset potential for oxidation is reached, gold is etched with chloride (Fig. 2(3), generating chloroauric acid in the process ${ }^{21}$ following the reaction ${ }^{24}$ :

$$
\mathrm{Au}+4 \mathrm{Cl}^{-} \rightarrow \mathrm{AuCl}_{4}^{-}+3 e^{-} \quad E^{0}=1.002 \mathrm{~V}
$$

This process is anisotropic, preferentially etching away gold at specific step edges, kinks, and vacancies, where the formation of chloride adlayers is more stable ${ }^{21}$, generating, in turn, more nucleation points for growing nanostructures. The freshly dissolved chloroauric acid quickly coordinates with free micellar $\mathrm{CTA}^{+}$in solution, 133 preventing chloroauric acid from diffusing away from the surface. Switching the polarity 134 of the scan rate, we observe the micellar $\mathrm{CTA}-\mathrm{AuCl}_{4}$ being reduced and deposited on 
135 the surface of the electrode at $\sim 0.6 \mathrm{~V}$ (Fig. 2-(4)), an overpotential required because of the formed CTA ${ }^{+}$layer on gold, following reaction (1) in reverse order.

137 Reduction of the CTA-AuCl 4 proceeds anisotropically, preferentially on the gold

138 facets where the CTA ${ }^{+}$layer is less dense, the surface energy is lower, and the edges 139 of the crystalline faces increase the curvature. Thus, access to micelles is easier, 140 increasing the reduction rate of gold species, promoting more efficient growth ${ }^{25,26}$ (Fig.

141 2-(4)). Iteration of this process yields the formation of increasingly large surfaces, so

142 the cathodic current at this step is more significant after each cycle (Fig. 2 cyclic 143 voltammogram).

145 Electrochemical and antifouling properties of NSG

146 A method to calculate the gold surface area is the electrochemical oxidation of 147 gold followed by integrating the cathodic peak during electroreduction. Following this method, the SEEDING process achieved a roughness factor $>200$ (Fig. 3a).

149 The oxidation/reduction of an equimolar electroactive couple, potassium 150 ferrocyanide and potassium ferricyanide, exhibits a reversible redox reaction with 151 diffusion-limited kinetics in both electrodes but with increased current densities for 152 NSG than flat gold electrodes (Fig. 3b) This is caused by the morphology of the surface of the electrode, accounting for both the roughness ${ }^{27,28}$ and porosity ${ }^{29}$, which could enhance the apparent electrode kinetics and depend on the cumulative pore

155 volume and $\operatorname{size}^{30}$. At slow scan rates and in longer time-scale experiments, the 156 electrode kinetics are governed by larger diffusion layers. Therefore, the process is 157 diffusion-limited, and most of the electrodes present semi-infinite planar diffusion 158 layers similar to a flat electrode. However, at faster scan rates, where the diffusion 159 layers are smaller and become comparable to the size of the electrode nanostructures 
and nanopores, the enhanced electrode kinetics arise from the morphology of the electrodes (Fig. 3c).

The formation of oxide adlayers on polarised gold electrode surfaces exhibits crystalline face selectivity, that is, the voltammetric oxidation peak has different energy

164 for each crystalline facet orientation $A u\{100\}<A u\{110\}<A u\{111\}^{31-33}$. NSG electrodes prepared at $1.20 \mathrm{~V}$ oxidating potential show an increase in $\mathrm{Au}\{110\}$ compared to $A u\{100\}$ and Au\{111\} (Fig. 3d). Grazing incidence X-ray diffraction on the NSG electrodes revealed the expected peaks for polycrystalline gold indexed to diffraction from the $A u(111), A u(200), A u(220)$, and $A u(311)$ planes of the face-centred cubic (fcc) structure of metallic gold (JCPDS, card\# 04-0784). The NSG surfaces exhibit an increased peak intensity compared to the flat gold electrodes, which could be explained by the increased thickness of the NSG electrode (Fig. 3e). The mean size of the crystalline domain for NSG was $21 \pm 4 \mathrm{~nm}$ (Supplementary Table 1), comparable to the grain size observed with scanning electron microscopy (SEM) (Fig. 1d, Supplementary Fig. 3) but with different facet relative abundances (Supplementary Table 1). The crystalline orientation of $A u\{110\}$ was the most abundant in both cases, flat gold and NSG, in agreement with previous electrochemical measurements (Fig. 3d). However, the relative abundance of $A u\{100\}$ and $A u\{111\}$ facet formation was larger in NSG than in flat gold (Supplementary Table 1). Additionally, the relative abundance of $A u\{111\}$ and $A u\{100\}$ is small compared to the abundant $A u\{110\}$ peak, probably because the interatomic gold distance of this facet opens a cavity large enough to accommodate $\mathrm{CTA}^{+}$cationic heads ${ }^{25}$.

183 To assess the anti-biofouling performance of the NSG electrodes, we compared 184 the electron charge transfer resistance $\left(R_{c t}\right)$, that is, the resistance to transfer an 
electric charge between the electrode and a diffusible redox molecule in different media. Flat surfaces showed large adsorption of BSA increasing $R_{c t}>4000 \%$ of their original value, whereas NSG remained almost undisturbed during $20 \mathrm{~h}$ of exposure

(Fig. 3f). The same trend was observed in plasma, with the NSG displaying one order of magnitude less $R_{c t}$. The anti-biofouling capability of NSG is attributed to the limited diffusion of the biofouling proteins throughout the nanopore substrate ${ }^{15}$, effectively limiting absorption and preventing the blocking of available electroactive sites (Fig. $3 g)$.

\section{Applications of NSG electrodes for biosensing}

We tested NSG electrodes for biosensing and diagnostic applications using various analytes. First, we detected ferrocyanide, an electroactive redox molecule, to understand the fundamental analytical parameters of these substrates. Then, we designed an immunoaffinity biosensor functionalising the surface of the NSG electrode with an appropriate bioreceptor for the detection of IL6, a soluble protein inflammatory marker commonly found in plasma. A specially tuned detection strategy was used to exploit the potential of our NSG electrodes while avoiding biofouling from the plasma. Later, this detection strategy was translated to detect generic EVs through binding the transmembrane proteins such as CD9 and CD81. Finally, we analysed a small cohort of clinical samples from patients with prostate cancer, detecting minute concentrations of tEVs from undiluted blood plasma ${ }^{34}$.

The voltammetric detection of ferrocyanide (Fig. 4a) drives a redox reaction with rapid electron transfer compared with the diffusion of species towards the surface. The diffusion profile for this reaction on flat electrodes is planar, whereas on the irregular 
2106 to $14 \mu \mathrm{A} \mathrm{mm} \mathrm{mm}^{-1}$ (Fig. 4a). However, at low concentrations, these species do not

211 have enough time to diffuse within the entire porous substrate before the reaction

212 occurs, and the limit of detection (LOD) is marginally improved by using NSG

213 electrodes (from 0.3 to $0.1 \mathrm{mM}$ ), which is in agreement with findings of previous 214 studies $^{16,17}$.

215 Therefore, we designed a detection strategy for our biosensor based on the 216 formation of an electroactive adsorbate compound that precipitates on the surface of 217 the electrode during the detection process. In this scenario, the kinetics of the 218 detection (oxidation of the mediator) is limited by the electron transfer of the mediator 219 to the surface of the electrode, rather than its diffusion ${ }^{16,17}$. This strategy was tested 220 using a selective antibody-functionalised NSG electrode biosensor to detect IL6. The 221 protein detection was conducted by an ELISA on the electrodes, generating an 222 electroactive precipitate at the end of the assay, which yielded a large current output 223 proportional to the concentration of IL6 (Fig. 4b). This allowed us to conduct more 224 sensitive measurements, achieving a 17-fold higher sensitivity with an LOD of 1 pg $225 \mathrm{~mL}^{-1}$ for NSG (vs. $31 \mathrm{pg} \mathrm{mL}^{-1}$ for flat electrodes) (Fig. 4c). Moreover, the critical feature 226 was the robustness of the biosensor when conducted the same measurements in 227 human plasma. Although the flat electrodes were completely passivated and detection 228 of IL6 was not possible, the NSG electrodes showed only an increase of the LOD from 2291 to $10 \mathrm{pg} \mathrm{mL}^{-1}$. Surfaces in contact with a biological sample, such as plasma, quickly 230 adsorb proteins that generate a passivating multilayer. Furthermore, this process is 231 typically enhanced in nanostructured surfaces because a larger surface area is available. This is why enhancing the sensitivity of the assay by only increasing the 233 surface area with nanostructures is not beneficial. Instead, it yields an unexpectedly 234 low sensitivity with real samples. However, if the electrode also has a large 
nanoporous structure beneath the surface, the diffusion of these contaminating proteins into the sensing area and the passivation are limited ${ }^{15}$.

237 By harnessing the anti-biofouling and ultrasensitive detection capabilities of this 238 strategy, we employed the NSG-based electrochemical biosensor to detect tEVs in 239 undiluted blood plasma, which are promising biomarker candidates for minimally 240 invasive cancer diagnostics. Here, we functionalised the NSG electrodes with anti241 CD9, an EV-associated biomarker, to capture EVs on the surface and then used specific detection antibodies to investigate the presence of particular biomarkers on 243 the EV.

244 NSG biosensors could capture EVs from lymph node carcinoma of the prostate 245 (LNCaP) cell culture supernatant spiked human plasma and selectively detecting the biomarkers. We detected CD9 and CD81 for total EV quantification and EpCAM, a 247 tumour-associated antigen, for tEV quantification (Fig. 4d). NSG biosensors allowed 248 us to detect total EVs and tEVs with high sensitivity in $5 \mu \mathrm{L}$ of plasma, achieving a 249 LOD for total EVs (CD9 $\left.{ }^{+} E V s\right)$ of 60 vesicles $\mu L^{-1}$ and tEVs (EpCAM+EVs) of 300 250 vesicles $\mu \mathrm{L}^{-1}$. For comparison, flat gold electrodes were electrically passivated during 251 the assay in plasma, and the ELISA required $50 \mu \mathrm{L}$ of sample, achieving a LOD in PBS of 700 vesicles $\mu L^{-1}$ for total EVs and 8000 vesicles $\mu L^{-1}$ for tEVs (Supplementary. 253 Fig. 4a).

To investigate whether NSG biosensors could be used to detect tEVs in clinical samples, we analysed the concentration of $C D 9^{+} E V s$ and $E p C A M^{+} E V s$. EV signals in urine samples were drawn from a small cohort $(n=10)$ that included patients with prostate cancer (stages II, III, and IV), aged 54-82 years. Urine CD9EV levels were not significantly higher in patients with prostate cancer than in healthy controls (Fig. 
260 0.0001). Ultimately, we developed electrochemical biosensors that could operate in complex biological fluids such as undiluted blood plasma. Accordingly, we tested the blood plasma of a larger cohort $(n=18)$, which included patients with prostate cancer 263 (stages II, III, and IV), aged 66-82 years. EpCAM+EV levels were significantly higher 264 in patients with prostate cancer than in healthy controls (Fig. 4f). Receiver operating 265 characteristic (ROC) curves indicated that EV detection using single associated 266 marker CD9+EVs showed extensive overlap across the groups, with no discriminatory power for classifying patients with cancer vs. healthy controls (Fig. $\mathbf{4 g}$ ). Moreover, ELISA detection of either $C D 9^{+} E V$ s or EpCAM+EVs in the same clinical samples could not discriminate between healthy and cancer donors (Supplementary. Fig. 4b-c). Interestingly, EpCAM+EV levels measured by NSG biosensors in plasma were approximately 10 times higher than those in urine. However, both urine and plasma EpCAM+EV levels constituted good classifiers (AUC 0.91 and 0.90 , respectively) for differentiating patients with prostate cancer from healthy cases (Fig. $\mathbf{4 g}$ ). Finally, the serum PSA levels of all cancer cohorts (urine and plasma) differed widely, showing no correlation with prostate cancer stage $(p=0.7454)$ or Gleason sum $(p=0.6940)$. Therefore, these results indicated a correlation between circulating EpCAM+EV levels 277 in both urine and plasma and the presence of prostate cancer. Notably, these clinical samples were analysed directly with the NSG electrodes, without dilution or 279 preprocessing steps, suggesting the potential utility of this biosensing platform for 280 ultrasensitive detection of markers in complex matrices.

\section{Conclusions}

We developed SEEDING, a simple and fast $(1 \mathrm{~min})$ method to generate 
without templates. The high electroactive area achieved on these surfaces increased the sensitivity in bioassays because of the higher bioreceptor immobilisation yield.

287 Moreover, faster apparent electron transfer kinetics lead to amplification of the 288 electrochemical signals and porosity provided a size-exclusion mechanism that 289 prevented biofouling. This allowed direct exposure to biological fluids containing large 290 concentrations of contaminating proteins while maintaining high sensitivity.

Functionalising these surfaces with specific bioreceptors allowed the specific detection of diffusible proteins or EVs in low-volume complex matrices. Because the sensitivity of large surface electrodes is limited by the diffusion of species involved, we 294 took advantage of a transduction mechanism based on the precipitation of an electrochemical mediator upon completion of the assay. Notably, ultrasensitive 296 detection of tEVs from small volumes of undiluted plasma and urine samples allowed us to discriminate between healthy controls and patients with prostate cancer. SEEDING offers key advantages over traditional nanostructuring or nanoporous methods (Supplementary Table 2). Specifically, it is a relatively simple and fast process that works on photolithographic chips and generates nanostructured and nanoporous electrodes. The high sensitivity with anti-biofouling performance is a key aspect for analysing low-concentration biomarkers in real samples. Therefore, SEEDING may be used to develop quick, selective, sensitive, and miniaturised diagnostics in biological samples.

\section{References}

1. Heitzer, E., Haque, I. S., Roberts, C. E. S. \& Speicher, M. R. Current and future perspectives of liquid biopsies in genomics-driven oncology. Nat. Rev. Genet. 20, 7188 (2019).

2. van Niel, G., D'Angelo, G. \& Raposo, G. Shedding light on the cell biology of extracellular vesicles. Nat. Rev. Mol. Cell. Biol. 19, 213-228 (2018). 
3. Xu, R., et al. Extracellular vesicles in cancer - implications for future improvements in cancer care. Nat. Rev. Clin. Oncol. 15, 617-638 (2018).

4. Hoshino, A., et al. Extracellular vesicle and particle biomarkers define multiple human cancers. Cell. 182, 1044-1061 e1018 (2020).

5. Johnsen, K. B., Gudbergsson, J. M., Andresen, T. L. \& Simonsen, J. B. What is the blood concentration of extracellular vesicles? Implications for the use of extracellular vesicles as blood-borne biomarkers of cancer. Biochim. Biophys. Acta. Rev. Cancer. 1871, 109-116 (2019).

6. $\quad \mathrm{Li}, \mathrm{W}$., et al. Emerging nanotechnologies for liquid biopsy: The detection of circulating tumor cells and extracellular vesicles. Adv. Mater. 31, e1805344 (2019).

7. Yoshioka, Y., et al. Ultra-sensitive liquid biopsy of circulating extracellular vesicles using ExoScreen. Nat. Commun. 5, 3591 (2014).

8. Liang, K., et al. Nanoplasmonic quantification of tumor-derived extracellular vesicles in plasma microsamples for diagnosis and treatment monitoring. Nat. Biomed. Eng. 1, (2017).

9. Sabaté Del Río, J., Henry, O. Y. F., Jolly, P. \& Ingber, D. E. An antifouling coating that enables affinity-based electrochemical biosensing in complex biological fluids. Nat. Nanotechnol. 14, 1143-1149 (2019).

10. Soleymani, L., et al. Nanostructuring of patterned microelectrodes to enhance the sensitivity of electrochemical nucleic acids detection. Angew. Chem. Int. Edit. 48, 8457-8460 (2009).

11. Soleymani, L., Fang, Z., Sargent, E. H. \& Kelley, S. O. Programming the detection limits of biosensors through controlled nanostructuring. Nat. Nanotechnol. 4, 844 (2009).

12. Bin, X., Sargent, E. H. \& Kelley, S. O. Nanostructuring of sensors determines the efficiency of biomolecular capture. Anal. Chem. 82, 5928-5931 (2010).

13. Daggumati, P., Kurtulus, O., Chapman, C. A., Dimlioglu, D. \& Seker, E. Microfabrication of nanoporous gold patterns for cell-material interaction studies. J. Vis. Exp. e50678 (2013).

14. Erlebacher, J., Aziz, M. J., Karma, A., Dimitrov, N. \& Sieradzki, K. Evolution of nanoporosity in dealloying. Nature. 410, 450-453 (2001).

15. Patel, J., et al. Electrochemical properties of nanostructured porous gold electrodes in biofouling solutions. Anal. Chem. 85, 11610-11618 (2013).

16. Daggumati, P., Matharu, Z., Wang, L. \& Seker, E. Biofouling-resilient nanoporous gold electrodes for DNA sensing. Anal. Chem. 87, 8618-8622 (2015).

17. Daggumati, P., Matharu, Z. \& Seker, E. Effect of nanoporous gold thin film morphology on electrochemical DNA sensing. Anal. Chem. 87, 8149-8156 (2015).

18. Arroyo-Currás, N., Scida, K., Ploense, K. L., Kippin, T. E. \& Plaxco, K. W. High surface area electrodes generated via electrochemical roughening improve the signaling of electrochemical aptamer-based biosensors. Anal. Chem. 89, 12185-12191 (2017). 
19. Khan, Z., Singh, T., Hussain, J. I. \& Hashmi, A. A. Au(III)-CTAB reduction by ascorbic acid: Preparation and characterization of gold nanoparticles. Colloids Surf. B. 104, 1117 (2013).

20. Ramírez-Rico, D. S. \& Larios-Durán, E. R. Electrochemical study on electrodissolution of gold in acidic medium using chlorides as ligands. J. Electrochem. Soc. 164, H994H1002 (2017).

21. Ye, S., Ishibashi, C. \& Uosaki, K. Anisotropic dissolution of an $\mathrm{Au}(111)$ electrode in perchloric acid solution containing chloride anion investigated by in situ STM - The important role of adsorbed chloride anion. Langmuir. 15, 807-812 (1999).

22. Vivek, J. P. \& Burgess, I. J. Quaternary ammonium bromide surfactant adsorption on low-index surfaces of gold. 1. Au(111). Langmuir. 28, 5031-5039 (2012).

23. Vivek, J. P. \& Burgess, I. J. Quaternary ammonium bromide surfactant adsorption on low-index surfaces of gold. 2. $\mathrm{Au}(100)$ and the role of crystallographic-dependent adsorption in the formation of anisotropic nanoparticles. Langmuir. 28, 5040-5047 (2012).

24. Weast, R. C., Astle, M. J. \& Beyer, W. H. CRC Handbook of Chemistry and Physics, vol. 84. (CRC Press LLC, Boca Raton, FL, 2004).

25. Chhatre, A., Thaokar, R. \& Mehra, A. Formation of gold nanorods by seeded growth: mechanisms and modeling. Cryst. Growth Des. 18, 3269-3282 (2018).

26. Meena, S. K., et al. The role of halide ions in the anisotropic growth of gold nanoparticles: a microscopic, atomistic perspective. Phys. Chem. Chem. Phys. 18, 13246-13254 (2016).

27. Zuo, X., Xu, C. \& Xin, H. Simulation of voltammogram on rough electrode. Electrochim. Acta. 42, 2555-2558 (1997).

28. Menshykau, D., Streeter, I. \& Compton, R. G. Influence of electrode roughness on cyclic voltammetry. J. Phys. Chem. C. 112, 14428-14438 (2008).

29. Menshykau, D. \& Compton, R. G. The influence of electrode porosity on diffusional cyclic voltammetry. Electroanal. 20, 2387-2394 (2008).

30. Punckt, C., Pope, M. A. \& Aksay, I. A. On the electrochemical response of porous functionalized graphene electrodes. J. Phys. Chem. C. 117, 16076-16086 (2013).

31. Hersbach, T. J. P., Mints, V. A., Calle-Vallejo, F., Yanson, A. I. \& Koper, M. T. M. Anisotropic etching of rhodium and gold as the onset of nanoparticle formation by cathodic corrosion. Faraday Discuss. 193, 207-222 (2016).

32. Jeyabharathi, C., Ahrens, P., Hasse, U. \& Scholz, F. Identification of low-index crystal planes of polycrystalline gold on the basis of electrochemical oxide layer formation. $J$. Solid State Electrochem. 20, 3025-3031 (2016).

33. Hamelin, A. Cyclic voltammetry at gold single-crystal surfaces. Part 1. Behaviour at low-index faces. J, Electroanal. Chem. 407, 1-11 (1996). 
422 34. Ni, J., et al. Role of the EpCAM (CD326) in prostate cancer metastasis and progression. 423 Cancer Metastasis Rev. 31, 779-791 (2012).

424

425 


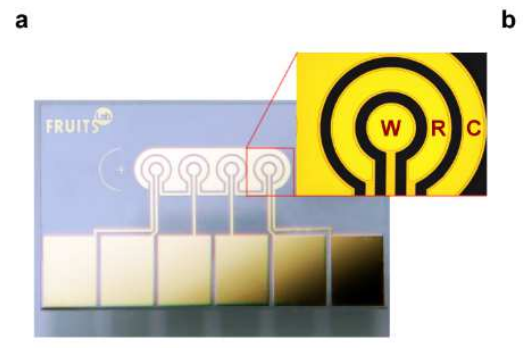

d

0 steps -0 s

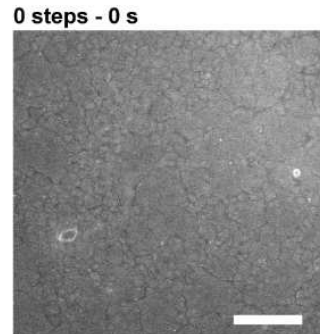

1,000 steps $-3 \mathrm{~s}$

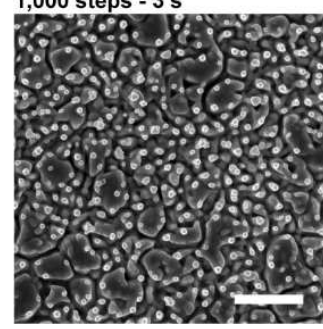

200 steps $-0.6 \mathrm{~s}$

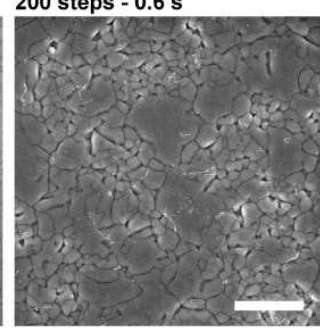

5,000 steps $-15 \mathrm{~s}$

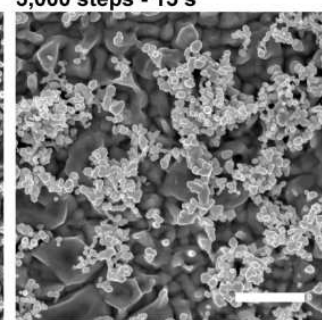

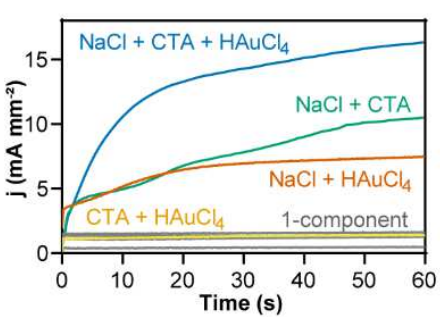

750 steps $-2.25 \mathrm{~s}$

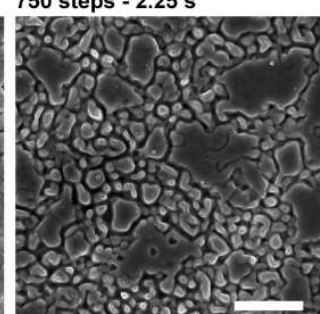

20,000 steps $-60 \mathrm{~s}$

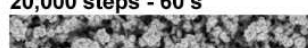

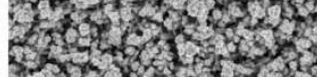
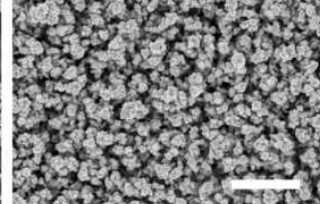
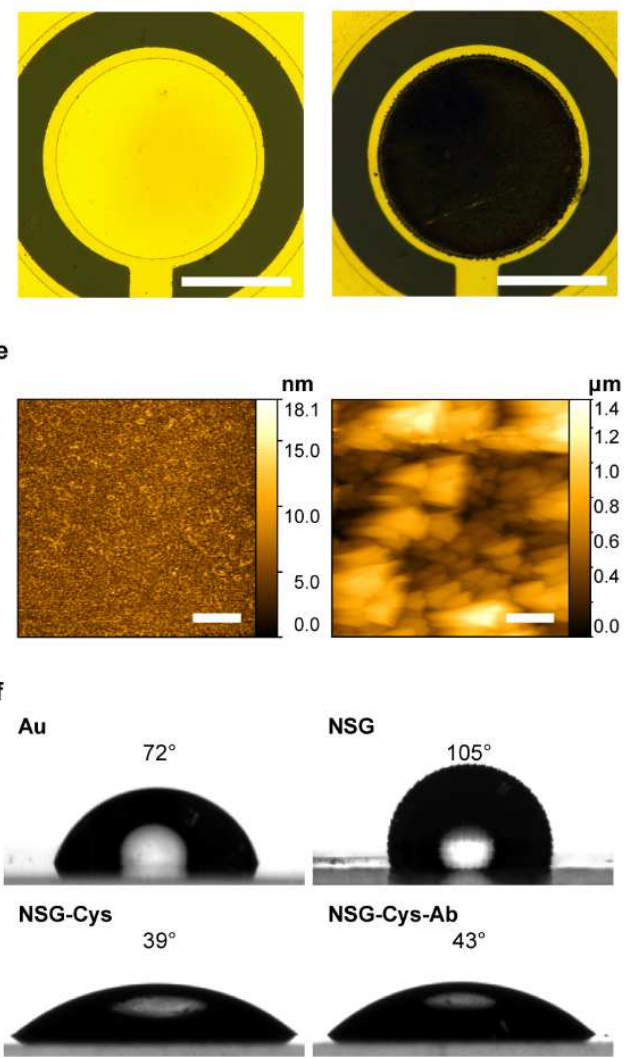

Fig. 1 | SEEDING of photolithographic flat gold electrodes. a, Photograph of a

429 photolithographic gold electrode chip with four circular working gold electrodes, a

430 common reference, and counter electrode. b, Typical chronoamperograms (only showing

431 anodic currents) during the SEEDING process conducted in photolithographic gold

432 electrodes in different control solutions (one-component controls are grayed out). c,

433 Photographs of the photolithographic gold electrodes before (left) and after (right) the

434 SEEDING process. Scale bar: $250 \mu \mathrm{m}$. d, Scanning electron micrographs of NSG

435 electrodes conducted by chronoamperometry after a different number of step voltages.

436 Scale bar: $1 \mu \mathrm{m}$. e, Atomic force microscopy topography of the photolithographic gold 437 electrodes before (left) and after (right) the SEEDING process. Scale bar: $1 \mu \mathrm{m} . \mathbf{f}$, Water 
438 contact angle photographs for different surfaces and modifications (Au: bare gold, NSG:

439 nanostructured and nanoporous gold, NSG-Cys: NSG modified with L-cysteine, NSG440 Cys-Ab: NSG-Cys functionalised with antibodies).

441 


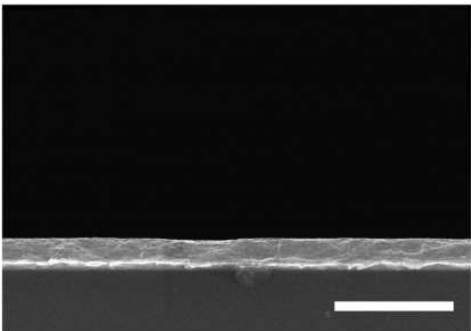

Flat gold surface

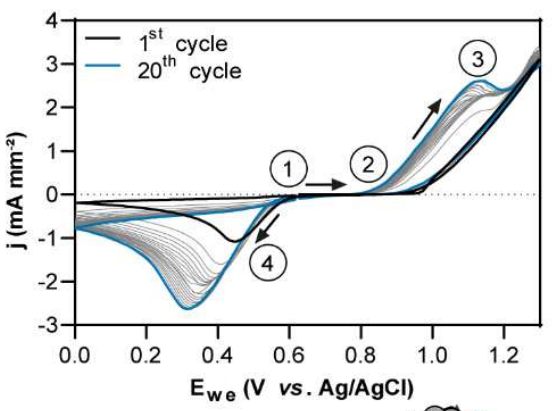

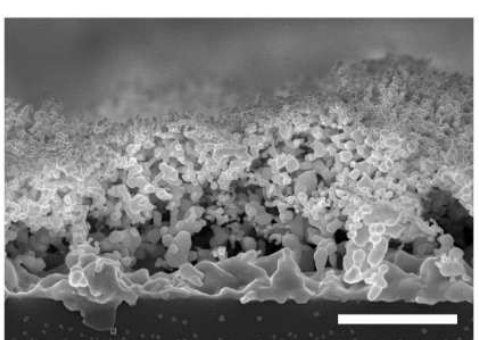

Nanostructured gold surface
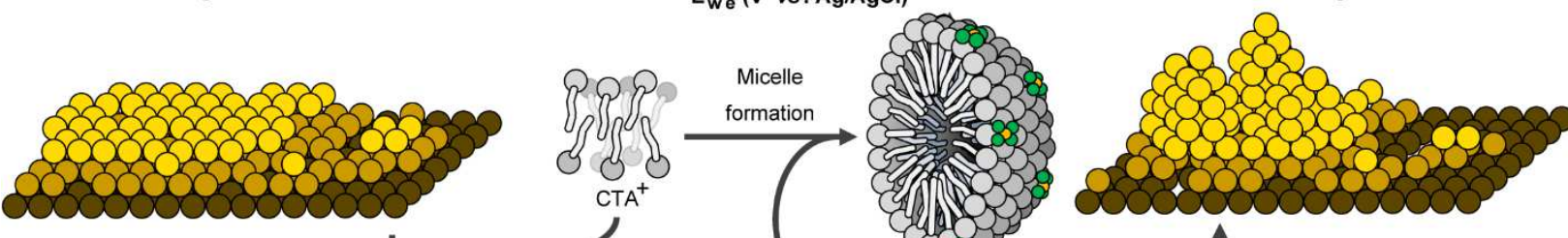

Adsorption of CTA to form a bilayer on gold

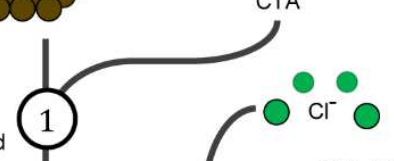

Chloride adlayer formation at

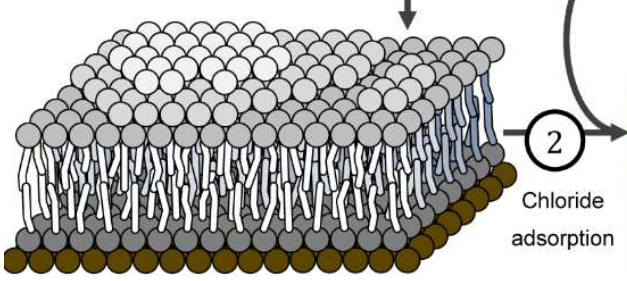

layer kinks, steps and vacancies
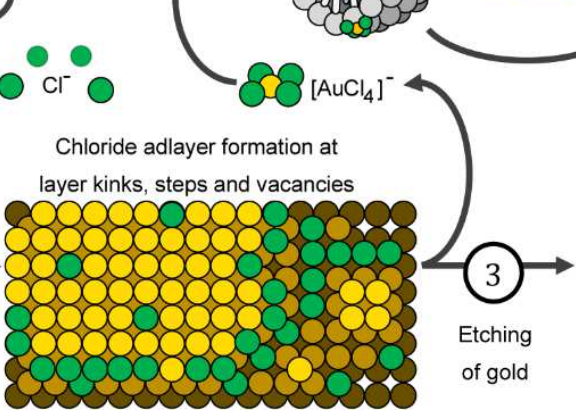

Fig. 2 | Schematic illustration of SEEDING. The SEEDING process is

electrochemically conducted in the presence of chloride, CTA ${ }^{+}$-based surfactant, and

chloroauric acid. (black: first scan; grey: subsequent scans; blue: last scan). (1),

447 Adsorption of CTA ${ }^{+}$takes place on gold, electrically passivating the surface of electrodes.

448 Additionally, CTA $^{+}$ions create micelles in the solution, which are loaded with negatively

449 charged chloroauric acid. (2), Electrochemically driven adsorption of chlorine adlayers on

450 gold step layers occurs, and (3), etching of the surface finally occurs, generating

451 chloroauric acid. (4), Electrochemically driven adsorption and reduction of gold-laden

$452 \mathrm{CTA}^{+}$micelles. The scanning electron micrographs show the cross-section of a 
453 photolithographic gold electrode before (left) and after (right) conducted the SEEDING 454 process. Scale bar: $1 \mu \mathrm{m}$.

455 
a

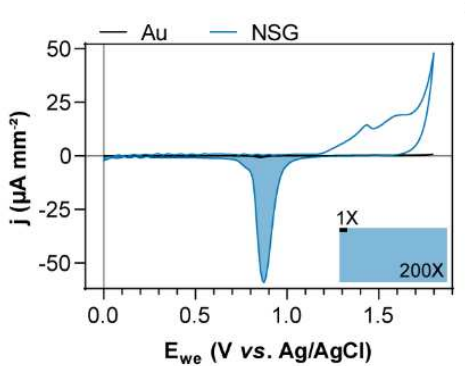

d

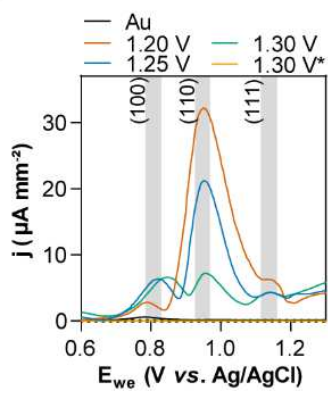

b
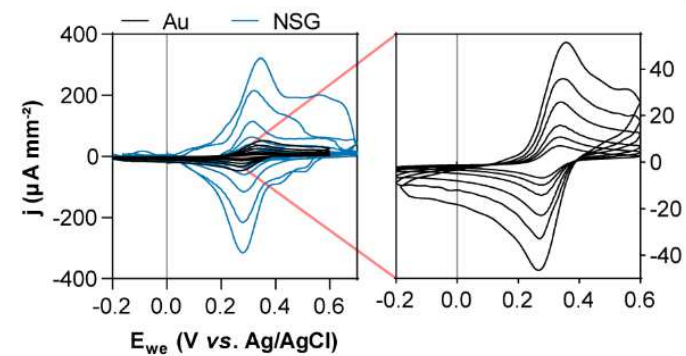

c

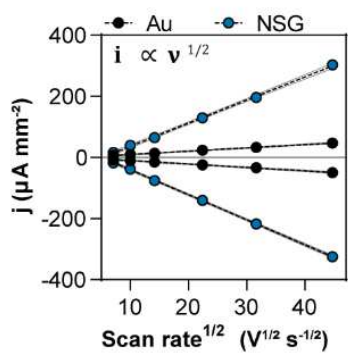

g

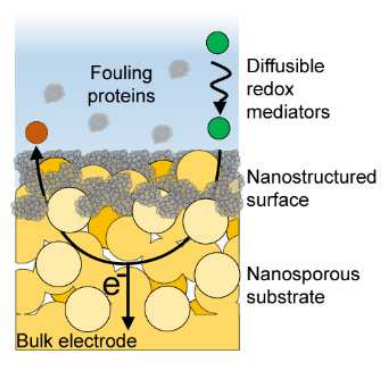

Fig. 3 | Electrochemical and antifouling properties of nanostructured and
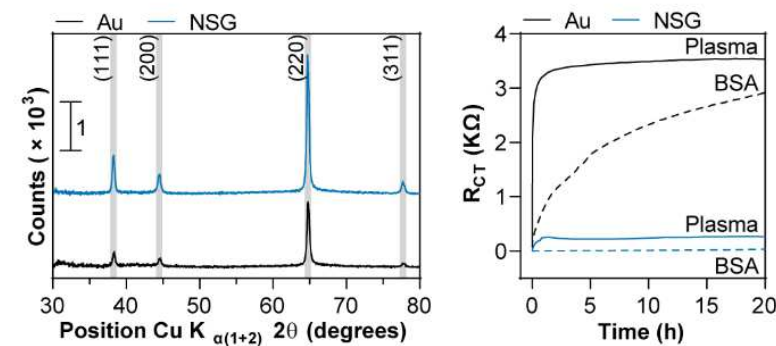

nanoporous gold. a, Cyclic voltammogram of a flat (black) and an NSG electrode (blue)

in acidic media (inset: schematic area enhancement). b. Cyclic voltammogram showing

oxidation and reduction peaks of an equimolar solution of $2.5 \mathrm{mM}$ ferri/ferrocyanide at

various scan rates for a flat gold electrode (black) and an NSG electrode (blue). The right

side plot is a magnified voltammogram of the flat electrode. c, Randles-Sevçik plot of

463 oxidation and reduction peak currents $\left(i_{p}\right)$ (circles) vs. the square root of the scan rate

464 (extracted from the voltammograms in $b)(n=4$ independent electrodes). Error bars

represent the standard deviation of the mean. $\mathbf{d}$, Square-wave scan voltammograms of

NSG electrodes prepared by chronoamperometry at different oxidation potentials vs. a

467 flat electrode as a control. (*no reduction step) e, Grazing incidence X-ray diffraction

468 spectrogram of an NSG electrode vs. a flat gold electrode. f, Plot of charge transfer 469 resistance change over time for a flat electrode (black lines) vs. an NSG electrode (blue 470 lines) in BSA $1 \%$ or human plasma (dashed lines). g, Schematic illustration of fouling 
471 mechanism on a porous electrode with pore size diameters smaller than the fouling 472 proteins.

473 


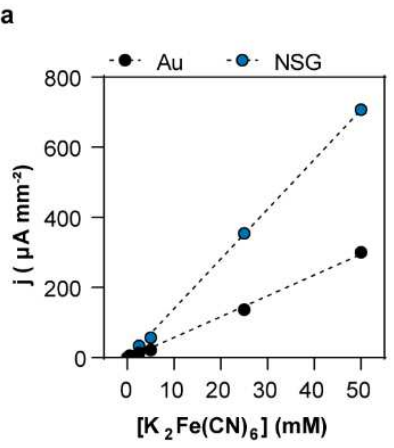

d

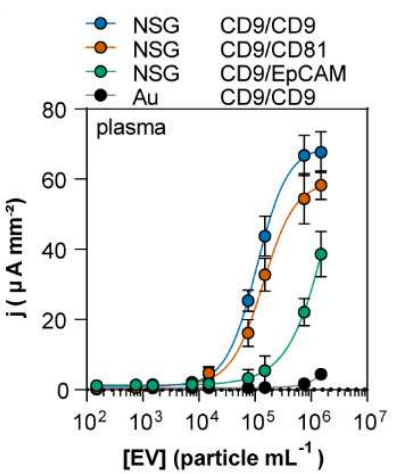

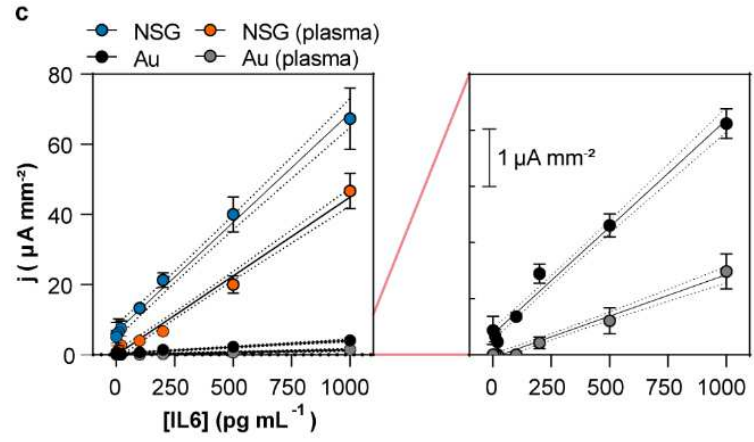

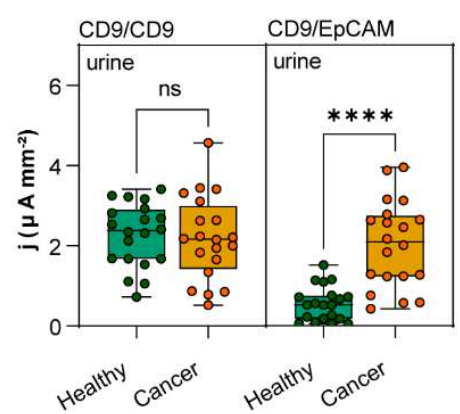

f

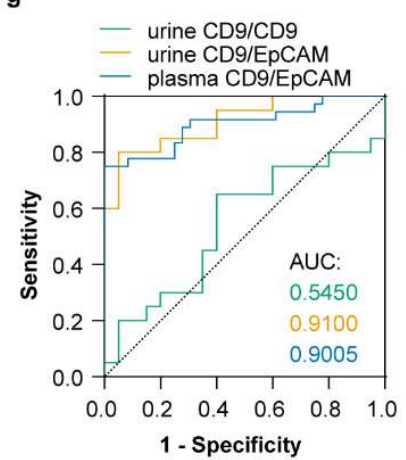

Fig. 4 | Application of nanostructured porous gold electrodes for analysis. a, Calibration plot representing peak current densities vs. concentration for the electrochemical detection of ferrocyanide on a flat electrode (black circles) vs. NSG electrodes (blue circles) ( $n=4$ independent electrodes). Error bars represent the standard deviation of the mean. b, Typical voltammetric oxidation peaks of precipitated electroactive reagent corresponding to a detection immunoassay on the NSG electrode chips. c, Calibration plot representing oxidation peak current (circles) recorded on NSG electrodes vs. flat electrodes (magnified on the right side) at different concentrations of IL6 spiked in PBS or human plasma ( $n=4$ independent electrodes). Error bars represent the standard deviation of the mean. $\mathbf{d}$, Calibration plot for $\mathrm{CD}^{+}, \mathrm{CD} 81^{+}$or $\mathrm{EpCAM}^{+}$on CD9-captured EV, representing peak current densities (circles) vs. particle concentration using NSG or flat electrodes ( $n=4$ independent electrodes). Error bars represent the 487 standard deviation of the mean. e, Analysis of clinical urine samples using NSG 
488 biosensors showing current density values (circles) with different assay schemes, 489 detection of $\mathrm{CD9}^{+}$or $\mathrm{EpCAM}^{+}$on CD9-captured $\mathrm{EV}$ ( $\mathrm{n}=20$ for each box, five biological 490 samples with four technical replicates for each). The boxes extend from the $25^{\text {th }}$ to $75^{\text {th }}$ 491 percentiles, the middle line is the median, and the whiskers extend from min to max values. 492 f, Electrochemical current density values (circles) for different clinical human plasma 493 samples from healthy and patients with cancer regarding the detection of $\mathrm{EpCAM}^{+}$on 494 CD9-captured EVs ( $n=36$ for each box, nine biological samples with four technical 495 replicates for each). The boxes extend from the $25^{\text {th }}$ to $75^{\text {th }}$ percentiles, the middle line is 496 the median, and the whiskers extend from min to max values. $\mathbf{g}$, ROC curves showing 497 the classification ability (healthy, cancer) for the three employed assays using either urine 498 or plasma clinical samples. 
Planar electrodes were fabricated in a cleanroom using standard lithography processes on 4-inch soda-lime glass wafers (Product \#1631, University wafers), 503 generating 44 chips $(1.5 \times 0.9 \mathrm{~mm})$ containing four working electrodes. First, $20 \mathrm{~nm}$ of 504 titanium and $400 \mathrm{~nm}$ of gold were deposited by e-beam evaporation (FC-2000, Temescal). 505 Following spin coating (4000 rpm, $500 \mathrm{rpm} \mathrm{s}^{-1}, 1 \mathrm{~min}$ ) of a $1.3 \mu \mathrm{m}$-thick photoresist (AZ® $5065214 \mathrm{E}$, MicroChemicals $\mathrm{GmbH}$ ), and evaporation of the solvent on a hot plate (90 s at $\left.507105^{\circ} \mathrm{C}\right)$, the wafer was exposed in a mask aligner (MA/BA6, SUSS MicroTec Korea Co. 508 Ltd.) (h-line $405 \mathrm{~nm}, 90 \mathrm{~mJ} \mathrm{~cm}^{-2}$ ) using a Mylar mask (Advance Reproductions, USA) 509 attached to a blank glass. After developing the photoresist (AZ® MIF 326 , 510 MicroChemicals $\mathrm{GmbH}$ ) to transfer the pattern, the exposed gold layer was etched with 511 a gold etchant (cat\# 651818-500ML, Sigma-Aldrich) for $1 \mathrm{~min}$, and the titanium layer was 512 stripped with a buffered oxide etchant (BOE 7:1, MicroChemicals $\mathrm{GmbH}$ ) for $40 \mathrm{~s}$. The 513 remaining positive photoresist was removed in isopropyl alcohol, and a $2 \mu$ m-thick 514 photoresist (SU-8 2002, K1 Solution) spin-coated on the wafer (3000 rpm, $500 \mathrm{rpm} \mathrm{s}^{-1}, 1$ $515 \mathrm{~min})$. Following a pre-baking step of the negative photoresist $\left(1.5 \mathrm{~min}\right.$ at $65^{\circ} \mathrm{C}$ and $3 \mathrm{~min}$ 516 at $95^{\circ} \mathrm{C}$ ), the wafers were exposed in a mask aligner (i-line $365 \mathrm{~nm}, 120 \mathrm{~mJ} \mathrm{~cm}^{-2}$ ) and 517 quickly post-baked $\left(1.5 \mathrm{~min}\right.$ at $65{ }^{\circ} \mathrm{C}$ and $1.5 \mathrm{~min}$ at $\left.95{ }^{\circ} \mathrm{C}\right)$ before developing the 518 photoresist (SU-8 developer, K1 Solution) to open up the electric contacts and limit the 519 sensing area on the electrodes. Finally, the chip was rinsed with isopropyl alcohol, and 520 the negative photoresist was further cured in a hard-bake step $\left(3 \mathrm{~h}\right.$ at $\left.180{ }^{\circ} \mathrm{C}\right)$. A protective 521 layer of positive photoresist was spin-coated to protect the chips during dicing and keep 
522 them clean during storage. Before use, the chips were rinsed in acetone and cleaned in

$523 \mathrm{O}_{2}$ plasma (Cute, Femto Science) at $0.5 \mathrm{mbar}$ and $50 \mathrm{~mW}$ for $2 \mathrm{~min}$.

Preparation of gold rod electrodes

Gold rod electrodes ( $\Phi=2 \mathrm{~mm}$, cat\# CHI101, Qrins) were prepared before use with

a polishing kit (cat\# CHI120, Qrins) by consecutively polishing their surfaces against

527 alumina slurries of different sizes on a polishing pad. First, we used a $1 \mu \mathrm{m}$ slurry on a

528 CarbiMet $^{\mathrm{TM}}$ disk, a $0.3 \mu \mathrm{m}$ slurry on a nylon pad, and finally, a $0.05 \mu \mathrm{m}$ slurry on a 529 microcloth pad using an "8"-shaped motion for approximately $30 \mathrm{~s}$, and sonicating the 530 electrode in a water bath (3510, Branson) for 1 min after each polishing step.

$531 \quad$ Preparation of SEEDING solution

532 The solution for SEEDING electrodes was prepared by dissolving $36 \mathrm{mg}$ of CTAC

533 (TCl, cat\# H0082, lot\# 4LL50-MN) per millilitre of a 222 mM sodium chloride stock solution.

534 Then, $1 / 9$ of the volume was added as chloroauric acid $10 \mathrm{mM}$ (stock stored in the dark 535 at $4{ }^{\circ} \mathrm{C}$ ) to obtain a final concentration of CTAC $100 \mathrm{mM}, \mathrm{NaCl} 200 \mathrm{mM}$, and $\mathrm{HAuCl}_{4} 1$ $536 \mathrm{mM}$. This solution was stable at room temperature $\left(25^{\circ} \mathrm{C}\right)$ when stored in the dark. 537 Preparation using CTAB (Sigma-Aldrich, cat\# 52365-50G, lot\# BCBT1510) generated 538 similar SEEDING performance, but the formulation was unstable over time.

$539 \quad$ Electrochemical setup and measurements

540 All the electrochemical processes and measurements were conducted in a 541 potentiostat-galvanostat EC-Lab (VSP model with a low-current option, BioLogic, France) 542 using a three-electrode configuration, with an external platinum wire as the counter 543 electrode and a miniaturised leak-free $\mathrm{Ag} / \mathrm{AgCl}$ electrode as the reference electrode (cat\# 544 ET072-1, Qrins). We used either photolithographic circular gold electrodes $(\Phi=0.45 \mathrm{~mm})$ 
545 or gold rod electrodes $(\Phi=2 \mathrm{~mm})$ for the working electrodes, with in-house-built

546 connector boxes. For photolithographic electrodes, we attached laser-cut double-sided

547 adhesive tape (DFM 200 clear 150 POLY H-9 V-95, FLEXcon) with a rectangular shape

548 and a main channel in the centre to generate a 5-10 $\mu \mathrm{L}$ reservoir for incubation of

549 samples on the electrodes. For gold rod electrodes, we used the bottom of a tube as

550 electrochemical cell (cat\# CLS430828-500EA, Sigma-Aldrich).

$551 \quad$ Calculation diffusion of species

552 The distance that electroactive diffusing particles moved away in one dimension from

553 the surface of the electrode after a certain time, that is, the thickness of the diffusion layer,

554 could be calculated using the root-mean-square displacement equation ${ }^{35}$ :

555

$$
\delta=\sqrt{2 D t}
$$

556

where $D\left(\mathrm{~cm}^{2} \mathrm{~s}^{-1}\right)$ is the diffusion constant of the reacting species, and $t$ is the time

557 (s). Using the parameters $\mathrm{D}=9.0 \times 10^{-6} \mathrm{~cm}^{2} \mathrm{~s}^{-1} 36$ and $\mathrm{t}=0.001 \mathrm{~s}$, the maximum diffusion 558 layer thickness was estimated as $1.9 \mu \mathrm{m}$.

559 The aggregation number of a micelle, $N_{a g g}$, could be estimated ${ }^{37}$. The volume of the 560 hydrophobic tail is given by:

$$
V_{0}=27.4+26.9 n_{c} \AA^{3}
$$

562

563

With $\mathrm{n}$ being the number of carbon atoms in the tail.

564 and $n_{c}^{\prime}$ carbon atoms inside the chains could be approximated by:

565

$$
R=1.6+1.265\left(n_{c}+1\right)+0.421 n_{c}^{\prime} \AA
$$

566 Thus, with the volume of ${ }^{+} \mathrm{N}\left(\mathrm{CH}_{3}\right)_{3}$ being $170 \AA^{3}$ :

$$
N_{\text {agg }}=\frac{4 / 3 \pi R^{3}}{170+V_{0}}
$$


For $\mathrm{CTA}^{+}$we have $n_{c}=16, n_{c}^{\prime}=1$, such that the radius is $\mathrm{R}=23.5 \AA$ and the $N_{a g g}=$

569 87. However, considering the concentration of the electrolyte $[\mathrm{NaCl}]=0.2 \mathrm{M}$, we could

570 estimate a $N_{a g g}$ of $\sim 200$, which is also in agreement with findings of other studies $\left(N_{a g g}=\right.$ $571 \quad 75-170)^{38}$.

572 In this scenario, we could estimate the diffusion constant $D\left(\mathrm{~m}^{2} \mathrm{~s}^{-1}\right)$ of a CTA $\mathrm{A}^{+}$micelle 573 using the Stoke-Einstein equation:

$$
D=\frac{k_{B} T}{6 \pi \eta R_{0}}
$$

where $k_{B}$ is the Boltzmann constant $\left(1.38065 \times 10^{-23} \mathrm{~m}^{2} \mathrm{~kg} \mathrm{~s}^{-2} \mathrm{~K}^{-1}\right), \mathrm{T}$ is the 576 temperature $(\mathrm{K}), \eta$ is the viscosity of the medium $\left(\mathrm{Pa} \mathrm{s}=\mathrm{kg} \mathrm{m}^{-1} \mathrm{~s}^{-1}\right)$, and $R_{0}$ is the radius 577 (m). Considering $R_{0}=23.5 \times 10^{-9} \mathrm{~m}, \mathrm{~T}=298 \mathrm{~K}$, and $=0.00089 \mathrm{Kg} \mathrm{m}^{-1} \mathrm{~s}^{-1}$, we obtain $\mathrm{D}=$ $5781.0 \times 10^{-6} \mathrm{~cm}^{2} \mathrm{~s}^{-1}$. This diffusion constant is lower than that for free $\mathrm{AuCl}_{4}^{-}$; thus, the quick 579 formation of $\mathrm{AuCl}_{4}^{-}$-CTA micellar complexes diffuses $\sim 2 \%$ of the distance from their free 580 counterpart, favouring the reutilisation of etched gold in the SEEDING process.

$$
\text { SEEDING on photolithographic electrodes by step chronoamperometry }
$$
SEEDING on photolithographic gold electrode chips conducted in $10 \mu \mathrm{L}$ of SEEDING 583 solution, by step chronoamperometry, consisting of 20,000 cycles of step voltages, first $5841.2 \mathrm{~V}$ for $1 \mathrm{~ms}$ and then $-1.2 \mathrm{~V}$ for $2 \mathrm{~ms}$. Finally, a chronoamperometric step at $-1.2 \mathrm{~V}$ was 585 applied for $10 \mathrm{~s}$ to reduce gold oxides.

\section{SEEDING on rod electrodes by cyclic voltammetry}

587 SEEDING on rod electrodes was conducted in $5 \mathrm{~mL}$ of SEEDING solution, by cyclic 588 voltammetry, consisting of 20 cycles between $1.25 \mathrm{~V}$ and $0 \mathrm{~V}$, starting at $0 \mathrm{~V}$ and a scan 589 rate of $0.1 \mathrm{~V} \mathrm{~s}^{-1}$. Finally, a chronoamperometric step at $-0.45 \mathrm{~V}$ was applied for $10 \mathrm{~s}$ to 590 reduce gold oxides. 
$0.5 \mathrm{M}$ at a scan rate of $0.1 \mathrm{~V} \mathrm{~s}^{-1}$, integrating the area (charge) under the reduction curve

594 at $\sim 0.9 \mathrm{~V}$. The cathodic peak was proportional to the amount of gold being reduced;

595 therefore, it was proportional to the surface area. Assuming a specific charge transfer of

$596400 \mu \mathrm{C} \mathrm{cm}^{-2} 39,40$, it is possible to calculate the electrode area. By comparing it with the 597 geometric area we obtained the roughness factor.

\section{Surface and topography characterisation by SEM and AFM}

599 The topographic characterisation of the electrode surfaces was conducted using SEM

600 (S-4800, Hitachi High-Technologies) and AFM (DI-3100, Veeco). Before SEM 601 characterisation, the samples were sputter-coated with a $5 \mathrm{~nm}$ layer of gold (E-1045, 602 Hitachi). Imaging was performed at a 3-4 mm working distance at an accelerating voltage 603 of $7 \mathrm{kV}$. The NSG electrode roughness and morphology were characterised by AFM in 604 non-contact tapping mode using silicon AFM tips with a radius curvature $<10 \mathrm{~nm}$ and 605 aluminium reflex coating (300AL-G-10, Woomyoung Inc.).

$606 \quad$ Contact angle measurements

607 Surface energy analysis was performed by water contact angle measurements, we 608 drop-casted $1 \mu \mathrm{L}$ drops of water on different surfaces, and images were immediately 609 recorded. The images were analysed with ImageJ 1.53e, approximating the shape of the 610 profile droplets to a sphere using a seven-point manual selection procedure. The contact 611 angle was calculated using the following formula:

$$
\theta=2 \cdot \operatorname{atan}\left(\frac{2 h}{l}\right)
$$


where $\theta$ is the contact angle (rad), " $\mathrm{h}$ " is the height of the droplet, and "l" is the length

614 of the base of the drop.

$615 \quad$ Crystallite size from X-ray diffraction

$616 \quad$ NSG surfaces were prepared on diced chips $(1 \mathrm{~cm} \times 1.5 \mathrm{~cm})$ with evaporated Ti/Au

$617(20 / 400 \mathrm{~nm})$ on a glass wafer. An adhesive tape with a squared $4 \mathrm{~mm}$ side opening was

618 attached to the surface to generate a working electrode and cover the rest. The SEEDING

619 process was conducted following the same method used for the electrode chips (see

620 SEEDING on photolithographic electrodes by step chronoamperometry).

621 The samples were analysed by grazing incidence X-ray diffraction (XRD) (Bruker D8

622 Discovery) with a $\mathrm{Cu} \mathrm{Ka}_{(1+2)}$ radiation source, using an incident angle $\omega=1^{\circ}$, and a 623 goniometer with $2 \theta$ range of $30^{\circ}-80^{\circ}$.

624 The crystallite size was calculated from XRD spectra, fitting the peaks to a Gaussian 625 model with Fityk 1.3.1, using the full width at half maximum from peak $A u(220)$, and using 626 the Scherrer equation:

$$
\tau=\frac{K \lambda}{\beta \cos \theta}
$$

where $\tau$ is the mean size of the crystalline domain, $\mathrm{K}$ is the dimensionless shape

629 factor (0.94), $\lambda$ is the X-ray wavelength $(\lambda=1.5418 \AA), \beta$ is the line broadening (rad), and $630 \theta$ is the Bragg angle $\left({ }^{\circ}\right)$.

$631 \quad$ Electrochemical characterisation of gold crystalline faces

632 We used SEEDING on photolithographic gold electrodes but using different oxidation 633 voltages, namely $1.20 \mathrm{~V}, 1.25 \mathrm{~V}$, and $1.3 \mathrm{~V}$. An additional set of electrodes was prepared 634 at $1.3 \mathrm{~V}$, but the reduction step for $10 \mathrm{~s}$ at $-1.2 \mathrm{~V}$ was omitted. Formation of different 635 crystalline gold faces generated on NSG electrodes was characterised electrochemically 
636 in an acidic media of $\mathrm{H}_{2} \mathrm{SO}_{4} 0.5 \mathrm{M}$ by squared wave voltammetry, scanning from $0 \mathrm{~V}$ to

$6371.3 \mathrm{~V}$ at a scan rate of $0.02 \mathrm{~V} \mathrm{~s}^{-1}$ (pulse height $30 \mathrm{mV}$, pulse width $100 \mathrm{~ms}$, step height 4 $638 \mathrm{mV})$.

$639 \quad$ Biofouling behaviour on NSG electrodes

640 The biofouling behaviour of NSG surfaces was characterised by Faradaic

641 electrochemical impedance spectroscopy in two different media, BSA $1 \%$ or human 642 plasma, containing an equimolar concentration of ferrocyanide $\mathrm{K}_{4} \mathrm{Fe}(\mathrm{CN})_{6}$ and 643 ferricyanide $\mathrm{K}_{3} \mathrm{Fe}(\mathrm{CN})_{6} 2.5 \mathrm{mM}$. Measurements were performed from $0.1 \mathrm{MHz}$ to $0.1 \mathrm{~Hz}$, 644 at an amplitude of $5 \mathrm{mV}$ vs. an open circuit potential. The $\mathrm{R}_{\mathrm{ct}}$ of the NSG electrodes was 645 determined by fitting the data from Nyquist plots to a Randles equivalent circuit, in which 646 the Rs models the resistance of the solution; the constant phase element (non-ideal 647 capacitance) was used to model the double-layer capacitance $\left(\mathrm{C}_{\mathrm{dl}}\right)$, and, in parallel, the $648 \mathrm{R}_{\mathrm{ct}}$ and a Warburg element $\left(\mathrm{Z}_{\mathrm{w}}\right)$ modelled the diffusion of electroactive species in solution 649 (Supplementary Fig. 5).

$650 \quad$ Electrochemical detection of redox molecules

651 Electrochemical detection of ferro/ferricyanide at different concentrations (50, 25, 5, $6522.5,0.5,0.25,0.05,0.025,0.005,0.0025$ and $0 \mathrm{mM}$ ) was conducted on NSG electrodes 653 using squared wave voltammetry between $0 \mathrm{~V}$ and $0.6 \mathrm{~V}$ at a scan rate of $0.02 \mathrm{~V} \mathrm{~s}^{-1}$ (pulse 654 height $30 \mathrm{mV}$, pulse width $100 \mathrm{~ms}$, step height $4 \mathrm{mV}$ ) using flat gold electrode as a control. $655 \quad$ Electrode functionalisation with capture antibodies

656 The electrode chips were immersed in a fresh solution of L-cysteine $10 \mathrm{mM}$ for $24 \mathrm{~h}$ 657 under constant shaking, and rinsed in ultrapure water. A glutaraldehyde solution $2.5 \%$ 658 prepared in PBS $\mathrm{pH}=8$ was drop-casted on the surface of the chips and incubated for 30 
$659 \mathrm{~min}$, after which the chips were rinsed and dried. A $20 \mu \mathrm{g} \mathrm{mL} \mathrm{H}^{-1}$ of anti-IL6 solution (Thermo 660 Fisher Scientific, cat\# CHC1263, lot\# 172402) or anti-CD9 (BD Pharmingen, cat\# 555370, 661 lot\# 9014503) was prepared in PBS, and deposited on individual electrodes with a 662 hollowed ceramic needle $\left(\Phi_{\mathrm{i}}=350 \mu \mathrm{m}\right.$; LabNEXT, Inc., no. 007-350) and incubated 663 overnight at $4{ }^{\circ} \mathrm{C}$ in a water-saturated atmosphere. After incubation, the electrodes were 664 washed with PBS Tween $200.05 \%$ in a shaker for 30 min to remove unbound antibodies. 665 Then, $10 \mu \mathrm{L}$ of $1 \mathrm{M}$ ethanolamine (Sigma-Aldrich, cat\# E9508) in PBS, adjusted to $\mathrm{pH} 7.4$, 666 with $\mathrm{HCl}$, was drop-cast on each electrode and incubated at room temperature for $30 \mathrm{~min}$ 667 to neutralise the unreacted moieties. Finally, the chips were incubated in $1 \%$ BSA at room 668 temperature for $1 \mathrm{~h}$ before use.

\section{Cell culture of LNCaP cancer cells, EV isolation, and concentration}

670 The EVs for standards were extracted from LNCaP cell supernatant and enriched by 671 centrifugal force filtration inside a disc containing a $20 \mathrm{~nm}$ anodised aluminium oxide 672 membrane filter ${ }^{41}$. LNCaP cells were obtained from ATCC and cultured in Roswell Park

673 Memorial Institute medium (Gibco, Thermo Fisher Scientific) supplemented with 5\% Exo674 Free FBS (Systems Biosciences, Inc.) and 1\% antibiotics/antimycotics. The cells were 675 incubated at $37{ }^{\circ} \mathrm{C}$ with $5 \% \mathrm{CO}_{2}$ for $48 \mathrm{~h}$. The cell culture supernatant was collected, 676 centrifuged at $300 \times \mathrm{g}$ for $10 \mathrm{~min}$, and centrifuged at $2000 \times \mathrm{g}$ for $15 \mathrm{~min}$ to completely 677 remove dead cells and cellular debris. The supernatant was filtered through a $450 \mathrm{~nm}$ 678 filter.

$679 \quad$ Preparation of EV standards

680 Enriched EVs from LNCaP cells were used to prepare standards. EVs were 681 reconstituted in PBS to measure the particle count number by nanoparticle-tracking 
analysis (Nanosight NS500, Malvern Instruments) and spiked in PBS or human plasma samples at different dilution rates to prepare the calibration curves.

\section{Clinical samples}

The biospecimens and data from cancer patients used for this study were provided by the Biobank of Pusan National University Hospital, a member of the Korea Biobank

Network. The study protocol was reviewed and approved by the IRB of Pusan National University Hospital (IRB 1802-004-063). Plasma samples from healthy donors were obtained from volunteers at the Yeungnam University Medical Center (IRB 2018-04-011). The biospecimens were collected after written informed consent was received from all

691 subjects. Within $2 \mathrm{~h}$ of sample collection, urine was centrifuged at $500 \times \mathrm{g}$ for $10 \mathrm{~min}$ at $4{ }^{\circ} \mathrm{C}$ to remove cellular debris and stored at $-80{ }^{\circ} \mathrm{C}$ until analysis. Urine samples were 693 thawed for analysis and centrifuged at $2500 \times \mathrm{g}$ for $15 \mathrm{~min}$ at $4{ }^{\circ} \mathrm{C}$, and the precipitated 694 pellet was discarded. Blood samples, $3 \mathrm{~mL}$, each were collected in vacutainer EDTA collection tubes and processed within $2 \mathrm{~h}$ of collection. Samples were centrifuged at $300 \times$ $\mathrm{g}$ for $10 \mathrm{~min}, 2000 \times \mathrm{g}$ for $10 \mathrm{~min}$ at $4{ }^{\circ} \mathrm{C}$ to remove cells and debris, and stored at $-80{ }^{\circ} \mathrm{C}$ 697 before use.

\section{Electrochemical enzymatic sandwich detection using NSG electrodes}

The reagents and dilutions used for the IL6 detection assay, and the washing steps, 700 were all conducted in assay buffer (1\% BSA and 0.05\% Tween 20 in PBS). IL6 (Thermo 701 Fisher Scientific, cat\# CHC1263) was spiked at different concentrations in PBS and 702 human plasma, and $20 \mu \mathrm{L}$ was incubated on the electrode chips for $2 \mathrm{~h}$. The biotinylated

703 anti-IL6 detection antibody (Thermo Fisher Scientific, cat\# CHC1263) was diluted to $1 \mu \mathrm{g}$ $704 \mathrm{~mL}^{-1}$ and incubated on the chips for $1 \mathrm{~h}$. To complete the assay, horseradish peroxidase 
705 (HRP)-streptavidin (Thermo Fisher Scientific, cat\# CHC1263) was diluted 1:400 in assay 706 buffer and incubated for $30 \mathrm{~min}$. Enhanced One-Component HRP Membrane Substrate 707 (Sigma-Aldrich, cat\# T9455) was incubated on the chips for 1 min. After each of the 708 previously described steps, the electrode chips were washed in a shacking Petri dish with 709 assay buffer for $15 \mathrm{~min}$. The electrode chips were individually interrogated by cyclic 710 voltammetry at a scan rate of $0.1 \mathrm{~V} \mathrm{~s}^{-1}$ between -0.5 and $0.5 \mathrm{~V}$ vs. open circuit potential 711 using the internal counter and pseudo-reference electrode of the chips. Other calibration 712 curves were obtained under different conditions by spiking IL6 in PBS instead of plasma 713 and using flat lithographic gold electrode chips for comparison.

714 The same assay was used to detect EVs in cell culture media and plasma using CD9 715 capturing antibodies and biotinylated CD9 (Abcam, monoclonal, clone\# MEM-61, cat\# 716 ab28094, lot\# GR280437-1) at $1 \mu \mathrm{g} \mathrm{mL}{ }^{-1}$, biotinylated CD81 (LSBio, cat\# LS-C134650, 717 clone\# 1.3.3.22) at $1 \mu \mathrm{g} \mathrm{mL} \mathrm{L}^{-1}$ or biotinylated EpCAM (R\&D Systems, cat\# DY960, lot\# 718 1336382) at $1 \mu \mathrm{g} \mathrm{mL}^{-1}$ as detection antibodies. Tween 20 was removed from the assay 719 entirely to prevent disruption of the lipid membrane of EVs during the assays. The 720 standards were prepared by diluting concentrated EV samples extracted from LNCaP cell 721 culture supernatant or spiked into human plasma at different dilutions.

722 Clinical urine and plasma samples from healthy and prostate cancer patient donors 723 were analysed in the same way by directly incubating the samples on the chips without 724 any pretreatment.

725 Enzyme-linked immunosorbent assay

726 ELISAs were conducted in 96-well plates (cat\# 3364, Corning). All the volumes are 727 per well, washing twice with $200 \mu \mathrm{L}$ of $0.1 \%$ BSA in PBS after each step. First, $50 \mu \mathrm{L}$ of 
anti-CD9 antibody (BD Biosciences, cat\# 555370, clone M-L13) at $4 \mu \mathrm{g} \mathrm{mL} \mathrm{m}^{-1}$ was incubated overnight at $4{ }^{\circ} \mathrm{C}$. After blocking with $200 \mu \mathrm{L}$ of $1 \%$ BSA for $3 \mathrm{~h}, 50 \mu \mathrm{L}$ of sample was incubated for $2 \mathrm{~h}$. Then, $50 \mu \mathrm{L}$ of biotinylated anti-CD9 (Abcam, cat\# ab28094) or anti-EpCAM (R\&D systems, cat\# DY960) at $500 \mathrm{ng} \mathrm{mL}^{-1}$ was incubated for $1 \mathrm{~h}$. Finally, $50 \mu \mathrm{L}$ of (HRP)-streptavidin (diluted 1:500 in $0.1 \%$ BSA) was incubated for 30 min.

733 Following addition of $50 \mu \mathrm{L}$ of stop solution, the absorbance was measured at $450 \mathrm{~nm}$ (Infinite 200 PRO NanoQuant Microplate Readers from Tecan, Tecan).

Statistical analysis

Statistical analysis was performed with Prism 9.1.0 (GraphPad). An $\alpha=0.05$ (confidence level 95\%) was considered to be statistically significance, with $p$-values ${ }^{*} p<0.05,{ }^{* *} p<0.01,{ }^{* * *} p<0.001$, and ${ }^{* * * *} p<0.0001$, respectively. The data presented in Fig.

4e, Fig. 4f, and Supplementary Fig. 4b were analysed by a two-tailed unpaired parametric Welch's t-test (equal variance not assumed). ROC curves (Fig. $\mathbf{4 g}$ and

Supplementary Fig. 4c) were constructed using the Wilson/Brown method to calculate the confidence interval ${ }^{42}$.

\section{Data availability}

The data that support the plots within this paper and other findings of this study are available from the authors upon reasonable request.

35. Bard, A. \& Faulkner, L. Electrochemical Methods: Fundamentals and Applications. (John Wiley \& Sons, Inc., New Jersey, 2001).

36. Martín, H., et al. Growth mode transition involving a potential-dependent isotropic to anisotropic surface atom diffusion change. Gold electrodeposition on HOPG followed by STM. Langmuir. 13, 100-110 (1997). 
37. Roelants, E. \& De Schryver, F. C. Parameters affecting aqueous micelles of CTAC, TTAC, and DTAC probed by fluorescence quenching. Langmuir. 3, 209-214 (1987).

38. Haynes, W. M. CRC Handbook of Chemistry and Physics, vol. 96. (CRC Press, California, 2016).

39. Hoogvliet, J. C., Dijksma, M., Kamp, B. \& van Bennekom, W. P. Electrochemical pretreatment of polycrystalline gold electrodes to produce a reproducible surface roughness for self-assembly: A study in phosphate buffer pH 7.4. Anal. Chem. 72, 2016$2021(2000)$.

40. Cherevko, S., Topalov, A. A., Zeradjanin, A. R., Katsounaros, I. \& Mayrhofer, K. J. J. Gold dissolution: towards understanding of noble metal corrosion. RSC Advances. 3, 1651616527 (2013).

41. Woo, H.-K., et al. Urine-based liquid biopsy: non-invasive and sensitive AR-V7 detection in urinary EVs from patients with prostate cancer. Lab. Chip. 19, 87-97 (2019).

42. Brown, L. D., Cai, T. T. \& DasGupta, A. Interval estimation for a binomial proportion. Stat. Sci. 16, 101-133, 133 (2001).

\section{Acknowledgements}

The biospecimens and data of patients with prostate cancer used for this study were provided by the Biobank of Pusan National University Hospital, a member of the Korea Biobank Network.

This research was supported by funding from the Institute for Basic Science (No. IBSR020-D1). This work was performed in part at the Central Research Facilities of the Ulsan National Institute for Science and Technology.

\section{Author contributions}

Conception and experimental design were performed by J.S.R. and Y.-K.C. Experiments, data analyses, validation, and reproducibility were conducted by J.S.R. ELISA assays were performed by H.-K.W. J.-H.P. conducted cell cultures and clinical sample handling and storage. The biospecimens and corresponding data of prostate cancer patients were 
788 given by H.K.H. The plasma samples of healthy donors were provided by J.-R.K. J.S.R 789 and Y.-K.C discussed the results and contributed to writing the manuscript.

790

$791 \quad$ Competing interests

792 J.S.R and Y.-K.C are listed as inventors on patents describing this technology.

$793 \quad$ Additional information

$794 \quad$ Supplementary Information is available for this paper.

795 Correspondence and requests for materials should be addressed to Yoon-Kyoung 796 Cho.

797 


\section{Supplementary Files}

This is a list of supplementary files associated with this preprint. Click to download.

- 20211008SI.pdf 